\title{
Uniting creative applications and asset management systems: New opportunities for developers and DAM vendors
}

\begin{abstract}
Mike Wallen
is Senior Product Manager for Adobe Creative Suite where he is responsible for product definition of Version Cue, managing developer technologies and ensuring there is digital asset management (DAM) integration into the Creative Suite. He has specialized expertise in XML, metadata, content/workflow management, digital asset management and cross-media publishing. Previously, he was Product Manager for InDesign CS2, Adobe PageMaker and FrameMaker. He has been working for Adobe since 1998 and before that he worked at a small prepress service bureau in Charleston, South Carolina. He holds a Bachelor of Science from the College of Charleston in Charleston, South Carolina, and a master of business administration from The Citadel in Charleston, South Carolina.
\end{abstract}

Keywords: DAM, CMS, creative suite, Adobe, workflow, integration

Abstract With the proliferation of different media and publishing platforms, the need for DAM systems has never been stronger. Digital assets are an increasingly invaluable part of an organization's intellectual property, yet deploying DAM systems across multiple workgroups and promoting active, productive use of systems is an ongoing challenge. With the release of Adobe Creative Suite 3, Adobe has raised the bar on integration among its desktop creative software applications. What is somewhat less obvious to DAM vendors and developers is that the company has also boosted integration capabilities and opportunities for the content and asset management world. Adobe introduced the Version Cue CS3 open API and software development kit (SDK). These tools enable developers to integrate DAM and publishing systems with Adobe Creative Suite 3 applications. The result is that developers and DAM providers can create custom asset management and workflow solutions that help enterprises simplify their processes and increase productivity across multiple workgroups - all while allowing creative professionals to stay within their desktop applications. Journal of Digital Asset Management (2007) 3, 139-144. doi:10.1057/palgrave.dam.3650075

\section{A COMPLEX LANDSCAPE SIMPLIFIED}

Part of the challenge for creative professionals using existing DAM systems is that there are many steps involved, and asset management processes typically occur outside the users' normal creative flow. For instance, it may take several steps using a web-based DAM system to download a photo from the web, edit it in Adobe Photoshop and upload it again, making it tougher for staff to meet tight deadlines. Adobe Creative Suite 3 provides capabilities that reduce the number of steps for common design tasks - whether it involves creating a Smart Object in Photoshop or placing multiple files into InDesign — without leaving a familiar and consistent interface. Organizations want the same productivity gains when managing digital assets, and this creates a tremendous opportunity of providing integrated DAM solutions that are tied with users' desktop software.

To date, tailored, integrated solutions have been difficult to supply because developers have had to write plug-ins for individual creative applications, an approach that is costly and complex. With the Version Cue CS3 API, Adobe is helping to close the gap between content and asset management systems and the tools creative professionals use. 


\section{ADOBE IN THE DAM WORLD}

Adobe wants creative teams to succeed, and also wants to help DAM vendors and developers thrive by taking advantage of the popularity of Adobe software, where the bulk of today's content for print, web and interactive media is generated. With this in mind, Adobe offers several technologies that come as a standard part of Adobe Creative Suite and enables developers to create customized, powerful DAM systems.

First, the Extensible Metadata Platform (XMP) is a labeling technology that allows users to embed data about a file, known as metadata, into the file itself. With XMP, desktop applications and back-end publishing systems gain a common method for capturing, sharing and leveraging this valuable metadata - opening the door for more efficient job processing, workflow automation and rights management, among many other possibilities. With XMP, Adobe has taken the heavy lifting out of metadata integration, offering content creators an easy way to embed meaningful information about their projects and providing industry partners with standards-based building blocks to develop optimized solutions. By providing a standard, W3C-compliant way of tagging files with metadata across products from Adobe and other vendors, XMP is a powerful solution enabler. As an open-source technology, it is freely available to developers, which means that the user community benefits from the innovations contributed by developers worldwide.

Furthermore, XMP is extensible, meaning that it can accommodate existing metadata schemas; therefore, systems do not need to be rebuilt from scratch. A growing number of third-party applications already support XMP.

\section{A WINDOW INTO ASSETS}

Another core building block in the asset management toolset is Adobe Bridge CS3, a powerful, easy-to-use media manager for visual people that lets them easily organize, browse, locate and view creative assets on the desktop. It introduces XMP into workflows in a simple way that is palatable for creative staff, promoting metadata adoption and use. The newest version of Bridge also offers direct access to services such as Bridge Home for finding instructional content, Adobe Stock Photos and Adobe

Acrobat Connect for online collaboration (Figures 1 and 2).

For developers, Bridge CS3 is now more extensible, creating opportunities for other types of solutions that automate workflows and tie together the capabilities of Creative Suite 3

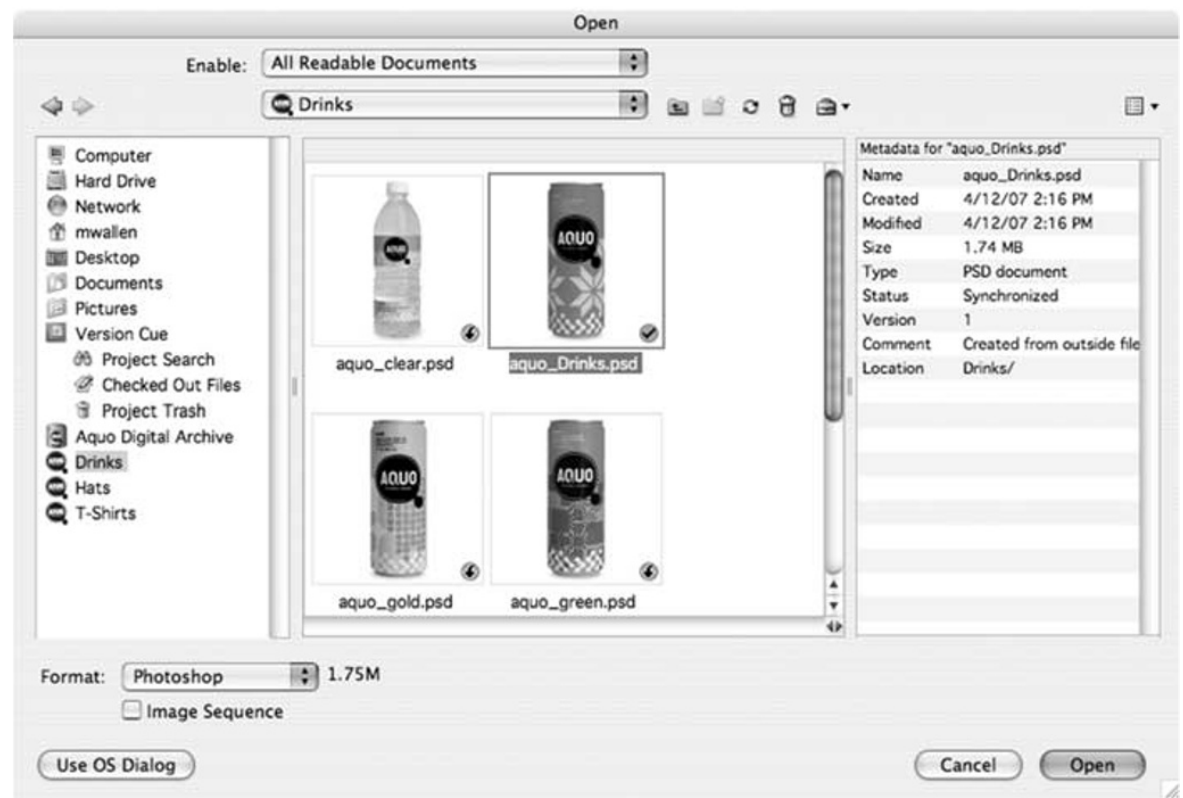

Figure 1: Integration with Adobe solutions would allow users to visually browse a DAM system directly from Photoshop, Illustrator, InDesign, InCopy, Bridge and Flash CS3 Professional 


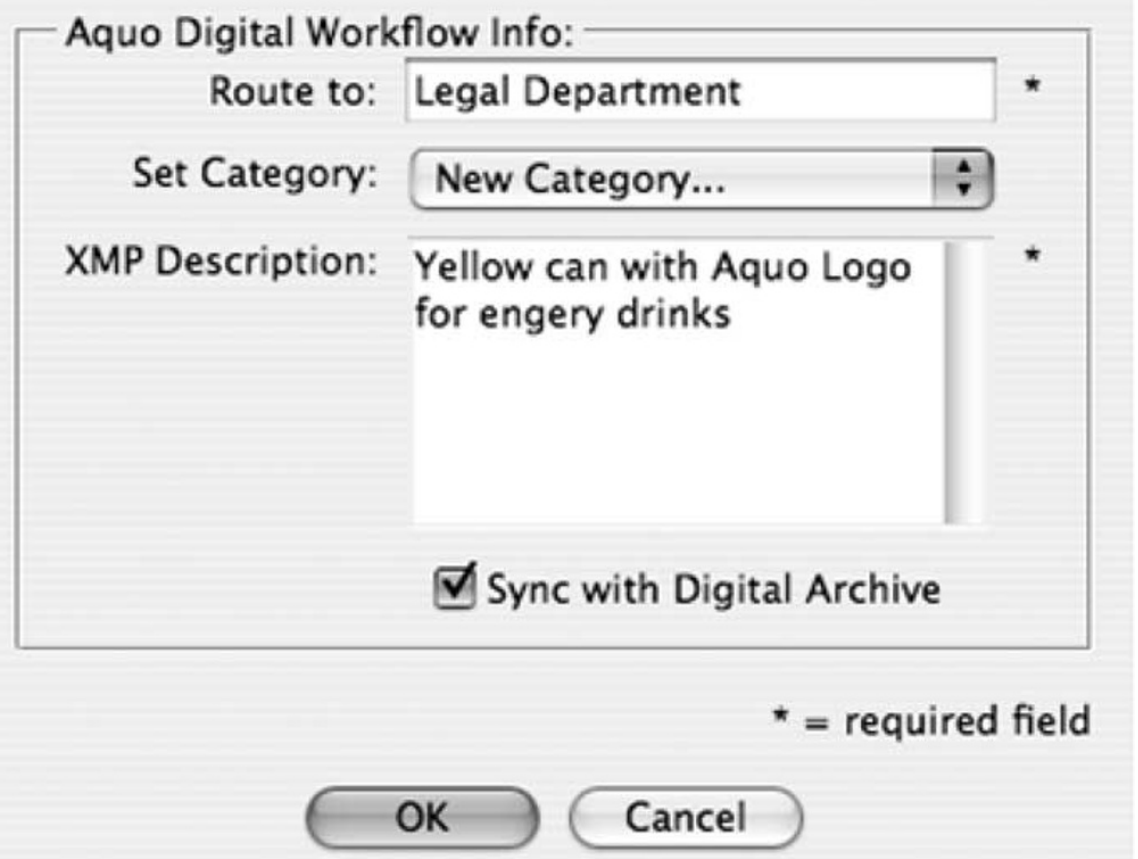

Figure 2: Using the latest tools from Adobe, developers could create solutions that require entry of metadata before an asset is checked into a DAM system

components. The JavaScript-based Bridge CS3 API can be used alone or together with the Version Cue CS3 API to automate processes. One solution might instruct Photoshop CS3 to generate a photo gallery and another might drive InDesign CS3 to create a contact sheet - all executed directly from within Bridge CS3. A developer could also create a panel with an interactive Flash application written in Flex.

\section{SIMPLE FILE MANAGEMENT FOR CREATIVE WORKGROUPS}

Another tool that workgroups can use to their advantage is Adobe Version Cue CS3, a serverbased file management system that is part of Adobe Creative Suite 3. With Version Cue CS3, users can check files in and out within shared projects, add comments and track file versions, perform XMP metadata searches, and host shared reviews of files. Version Cue is integrated with the components of Creative Suite 3 so that users can coordinate and manage projects and files without leaving their design environment.
Version Cue provides a centralized place for files and helps ensure that people are working on the latest versions (Figure 3).

New in Version Cue CS3 are powerful features for IT staff. Using an intuitive, web-based interface, IT can remotely configure servers and control access among users and groups for centralized project sharing and version control. Support for LDAP enables easy user account management and synchronization. IT staff can also easily manage security options, back up projects automatically on a recurring schedule, create projects by importing files from a folder or batch-delete old versions. The newest Version Cue also automatically broadcasts servers and shared projects to designers, without the need for complex network configuration (Figure 4).

\section{THE OPPORTUNITY FOR DEVELOPERS AND DAM VENDORS}

The Version Cue CS3 API and SDK are an attractive option for integration with enterprise DAM systems. Developers can use this new API to integrate their systems with the newest 


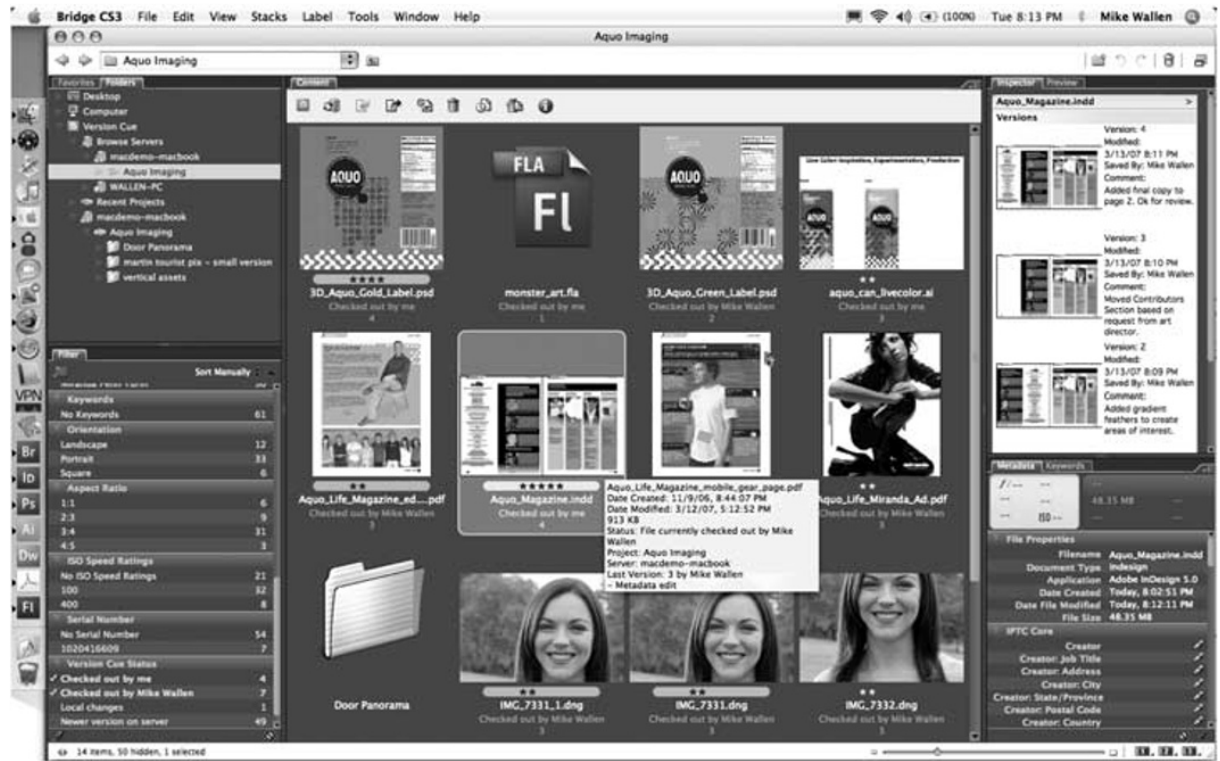

Figure 3: Version Cue makes it easy to see the status of a file, including the date created and modified

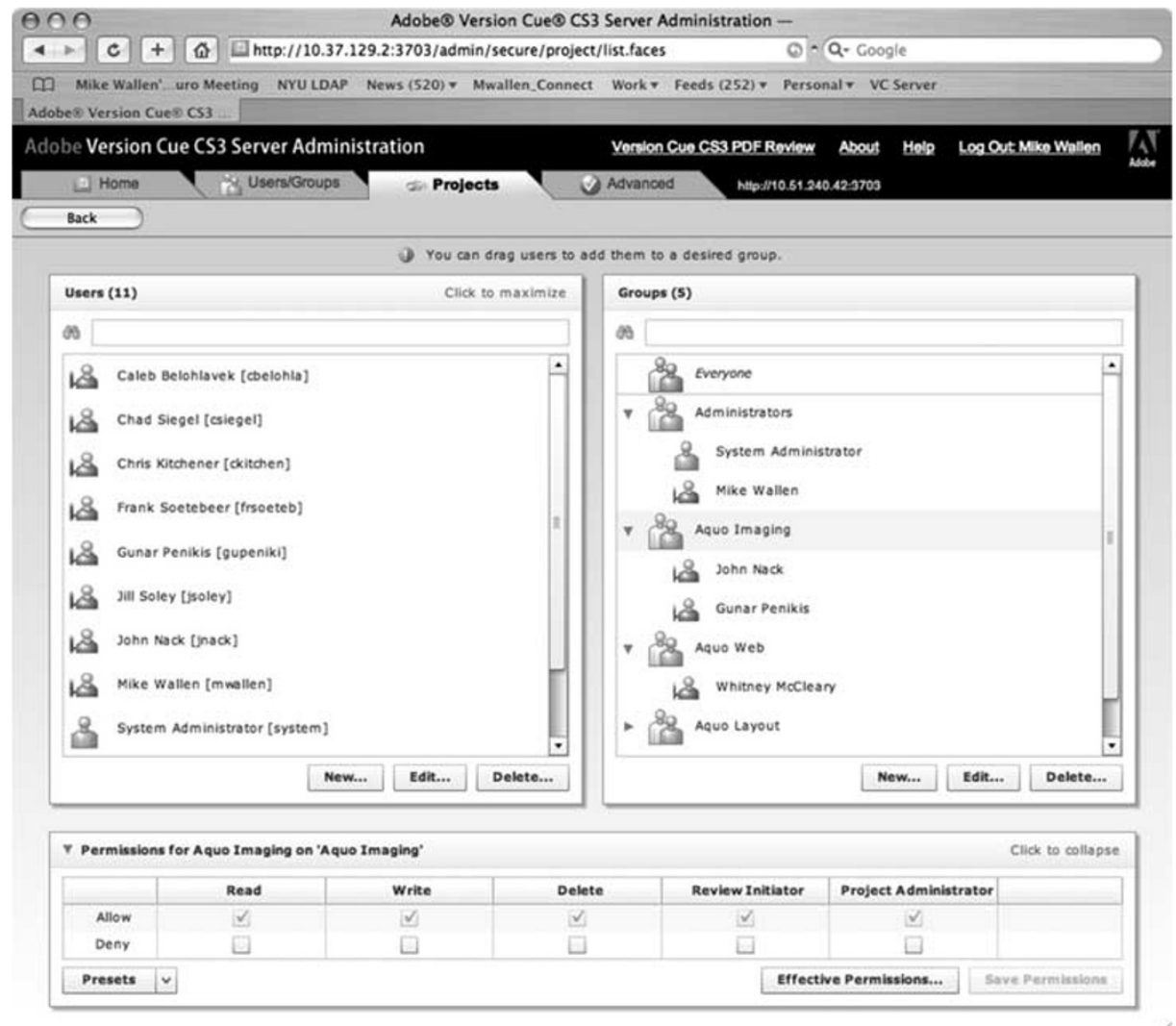

Figure 4: With Version Cue CS3, a web-based interface makes it easy for IT staffs to manage users and groups 
versions of Photoshop, Illustrator, InDesign, InCopy, Flash Professional and Bridge - without creating separate plug-ins. This common API represents a significant opportunity for DAM vendors, publishing system vendors and other developers to create customized solutions that are more functional for complex publishing environments and unique workflows.

The Version Cue API and SDK were designed with feedback from DAM and solution providers. The SDK is based on Eclipse, a familiar environment for Java and Flex developers. It enables integration with back-end systems into all of the Adobe Creative Suite 3 components and leverages the Version Cue CS3 infrastructure and user interface for application integration. Developers can take advantage of built-in capabilities of Version Cue and Bridge such as user authentication, file check-in and check-out or visual browsing of assets. The SDK also provides sample plug-ins and documentation covering the core use cases for asset management integration.

\section{MANY DIFFERENT OPPORTUNITIES}

Although Adobe Bridge and Version Cue CS3 can be used out of the box for basic solutions, there are several ways developers can take advantage of the Version Cue API to add functionality. Consider a workgroup that is not yet tapping into an existing enterprise DAM system to control and manage assets, but it is already using Adobe Creative Suite, including Version Cue and Bridge. A developer could create a plug-in to bring this workgroup onto the DAM system.

The Version Cue API enables creation of plug-ins for several different uses. For instance, DAM vendors or developers could create branded icons accessible from within Adobe Creative Suite 3 components to provide direct access to the DAM system. Or, a developer could deploy a Version Cue plug-in to provide authentication, controlling access to assets between Version Cue and the DAM system. Another use might be to more closely integrate the DAM system with Adobe Bridge, thereby enabling users to use the rich searching and browsing capabilities of Bridge to find assets contained in the DAM system. The developer could also enable display of custom metadata or add specialized search criteria for advanced queries. This is especially compelling considering that search is often the most prevalent way users access asset management systems. Another

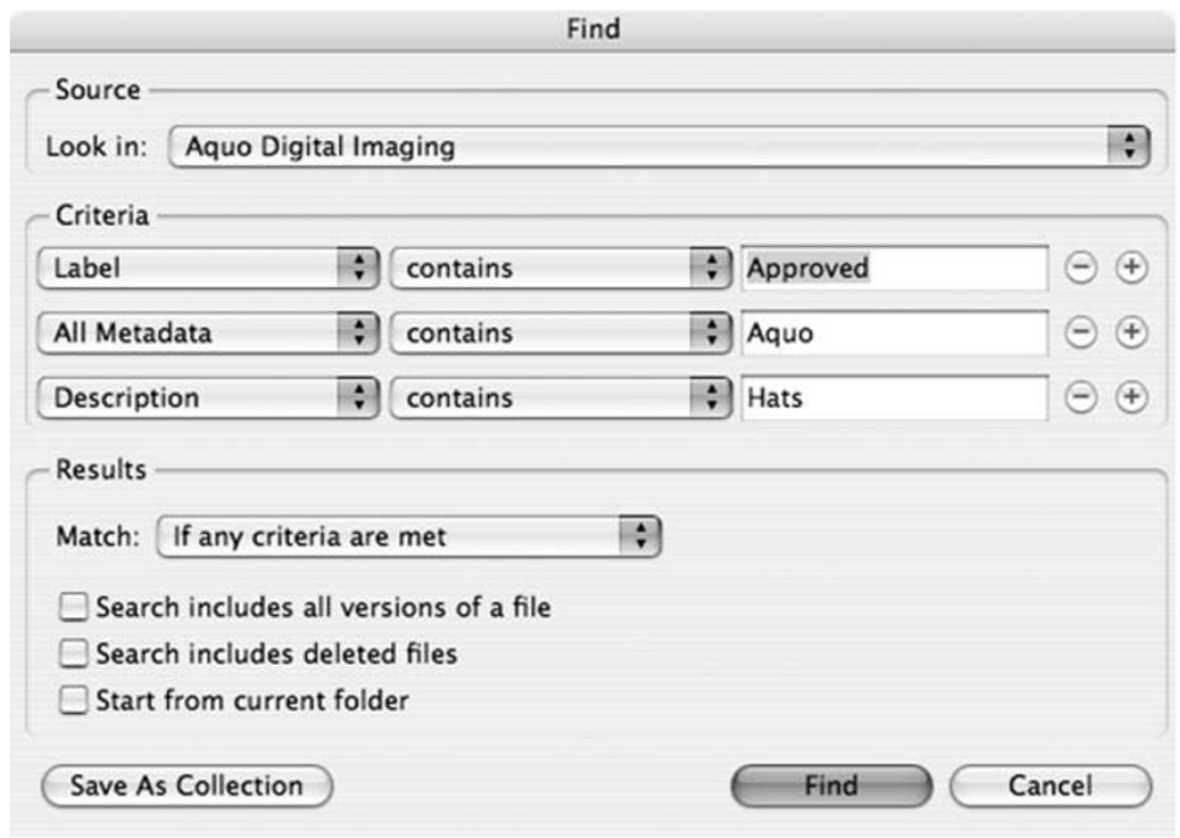

Figure 5: Searching is a key navigational aid when moving through systems with large volumes of assets. Custom search criteria can be added to support complex queries 
possible use for the Version Cue CS3 API might be to create a plug-in that allows users to double-click files contained in the DAM system and edit them directly in Adobe applications. File check-in and check-out would occur automatically (Figure 5).

\section{PLUG-INS IN THE WORKS}

Already, developers are taking advantage of the Version Cue CS3 API and other Adobe tools to create compelling, beneficial plug-ins.

Companies working with the Version Cue CS3 API and SDK to develop solutions include Adam, Extensis, NetXPosure and North Plains Systems.

Adobe worked with Ctrl-Publishing to provide sample plug-ins demonstrating common use cases of the Version Cue API. One example is a partial implementation of a JSR 170compliant connection to an Apache Software Foundation Jackrabbit content repository. The other sample is a plug-in that creates an RSS notification system accessible from Adobe applications. This plug-in could be used to notify users via RSS feed of events such as project check-in or status. These examples are designed to illustrate the enhancements and connectivity that can be developed.

With Creative Suite 3, Adobe is providing not only the desktop creative software but also a foundation of technologies that allow partners and developers - including DAM vendors - to break new ground and help customers succeed in ways that were not viable in the past.

The Version Cue CS3 API and SDK are available at no charge from http://www.adobe. com/devnet/versioncue/. A copy of the Version Cue Server, which comes for free with a licensed copy Adobe Creative Suite 3 Design and Web editions and Adobe Creative Suite 3 Master Collection, is required. 